\title{
Mediation of the Anticancer Effects of Resveratrol via the Upregulation of Tristetraprolin in Gastric Cancer Cell
}

\author{
Won-Tae Kim ${ }^{1 \#}$, Hua Jin", Se-Ra Lee ${ }^{1,3 \#}$, Sung Zoo Kim², Sun-Hee Leem ${ }^{1 *}$ and Soo Mi Kim \\ ${ }^{1}$ Department of Biological Science, Dong-A University, Busan 49315, Republic of Korea \\ ${ }^{2}$ Department of Physiology, Institute of Medical Science, Chonbuk National University Medical School, Jeonju, Republic of Korea \\ ${ }^{3}$ Division of Drug Development Optimization, Osong Medical Innovation Foundation (KBio), Chungbuk 28160, Republic of Korea \\ \#These authors contributed equally to this work
}

\begin{abstract}
Tristetraprolin (TTP) is an mRNA-binding protein that can induce mRNA degradation. It controls cell growth and factors related to tumor development by binding AU-rich elements located in the $3^{\prime}$ untranslated region (UTR) of mRNA, leading to rapid mRNA decay. Resveratrol, which is a natural flavonoid polyphenolic compound, has been shown to exert biological activities on various human cancers. However, there are no studies assessing its effects with TTP regulation on human gastric cancer. The present novel study demonstrated that the tumor suppressing effects of resveratrol were mediated via the induction of TTP expression in gastric cancer. We found that resveratrol induced TTP mRNA and protein expression in the gastric cancer cell lines SNU484 and SNU638. Treatment with resveratrol enhanced the decaying activity of TTP on luciferase mRNA containing cIAP2, LATS2, Lin28, and E2F1 in their 3' UTR. We also confirmed that TTP was upregulated. Target genes of TTP (E2F1, LATS2, and Lin28) were downregulated by resveratrol treatment in SNU484 cells as observed in a microarray experiment. Furthermore, we observed that resveratrol affects cell proliferation, metastasis, and tumor formation in SNU484 and SNU638 cells. Overall, the results of the present study suggest that resveratrol induced the mRNA and protein levels of TTP by regulating the decay of clAP2, LATS2, Lin28, and E2F1 mRNA. It can thus be concluded that resveratrol can be utilized as a potential therapeutic anticancer agent for treating human gastric cancer
\end{abstract}

Keywords: Tristetraprolin; Resveratrol; Gastric cancer; Proliferation; Metastasis

\section{Introduction}

Gastric cancer is one of the most fatal cancers and is the second leading cause of cancer-related deaths worldwide [1-4]. The highest incidence of and mortality rates due to gastric cancer are found in eastern and western Asian countries including South Korea. Moreover, the incidence of gastric cancer in South Korea has been observed to be $60-64 \%$ higher than in Asian countries with the highest incidence $[4,5]$. Since the mid- $20^{\text {th }}$ century in Asian countries, gastric cancer incidence and mortality rates have been steadily decreasing because of the declining prevalence of Helicobacter pylori infection; however, the corresponding rates have been increasing in the United States of America and many Western countries due to increasing prevalence of obesity [4,5]. Hence, developing a new classification system for gastric cancer and understanding the molecular pathogenesis of gastric cancer are required for to develop effective therapeutic approaches. In this way, a promising new method could be established that may help further reduce gastric cancer incidence and mortality rates. Therefore, it is urgent to identify novel therapeutic targets and preventive treatments to control the high mortality rates due to gastric cancer.

Recent studies have investigated a mechanism for the regulation of oncogenes at the post-transcriptional level through the tristetraprolin (TTP) family of CCCH tandem zinc finger protein (ZFP) [6-8]. The TTP family of proteins consists of three well-characterized members in mammals (ZFP36 or TTP, ZFP36L1 or TIS11B, and ZFP36L2 or TIS11D) and a fourth member in mice or rats but not in humans (ZFP36L3) [9-12]. TTP is an AU-rich element (ARE)-binding protein that mediates the decay of ARE-containing mRNAs such as those encoding inflammatory cytokines and proto-oncogenes [11,1315]. In previous studies, TTP negatively regulated the expression of cIAP2, LATS2, Lin28, E2F1, and other target genes [16-20]. This observation demonstrates a possible role for TTP in tumor growth and development. Further, mRNAs encoding TNF- $\alpha$ and GM-CSF have been stabilized in TTP knockout mice and in cells derived from them [21]. Moreover, the upregulation of TTP has been observed to cause significant decrease in TTP target gene expression and inflammatory responses in several cancer and immune cells [22-24]. These results supported the hypothesis that TTP is a potent tumor suppressor and an anti-inflammatory protein.

The possibility of natural agents inhibiting tumorigenesis has attracted the widespread attention in cancer prevention and treatment. The natural agent resveratrol (trans-3,4',5-trihydroxystibene) has been detected in more than 70 plant species, including grapes, berries, plums, peanuts, pines, and other plants $[25,26]$. It has been reported to possess antioxidant, anticancer, antiplatelet aggregation, and antiinflammatory properties $[26,27]$. There are several recent reports that have shown the ability of resveratrol to suppress cancer cell growth either alone or in combination with various anticancer drugs/ phytochemicals as well as to suppress growth factor-related cancer development [2833]. These results showed that resveratrol regulates cell proliferation, invasion, and metastasis of cancer cells by controlling several signaling pathways. In addition, resveratrol reduces the expression of multiple cytokines in breast cancer cell lines and promotes TTP expression at the

*Corresponding authors: Soo Mi Kim, DVM, Department of Physiology, Chonbuk National University Medical School, Gungiro 20, Deokjin-Gu, Jeonju 54907, Republic of Korea, Tel: +82-63-270-3077; E-mail: soomikim@jbnu.ac.kr

Sun-Hee Leem, Lab of Functional Genomics, Department of Biological Science, Dong-A University, 37 Nakdong-Daero 550-Beon-gil, Saha-Gu, Busan 49315, Republic of Korea, Tel: +82-51-200-5639; E-mail: shleem@dau.ac.kr

Received March 13, 2018; Accepted March 15, 2018; Published March 20, 2018

Citation: Kim WT, Jin H, Lee SR, Kim SZ, Leem SH, et al. (2018) Mediation of the Anticancer Effects of Resveratrol via the Upregulation of Tristetraprolin in Gastric Cancer Cell. Med Chem (Los Angeles) 8: 029-037. doi: 10.4172/2161 0444.1000494

Copyright: (c) $2018 \mathrm{Kim} \mathrm{WT}$, et al. This is an open-access article distributed under the terms of the Creative Commons Attribution License, which permits unrestricted use, distribution, and reproduction in any medium, provided the original author and source are credited. 
Citation: Kim WT, Jin H, Lee SR, Kim SZ, Leem SH, et al. (2018) Mediation of the Anticancer Effects of Resveratrol via the Upregulation of Tristetraprolin in Gastric Cancer Cell. Med Chem (Los Angeles) 8: 029-037. doi: 10.4172/2161-0444.1000494

mRNA and protein levels in a dose- and time-dependent manner [34]. Resveratrol has been also been reported to increase TTP expression in U87MG human glioma cells with destabilized urokinase plasminogen activator and urokinase plasminogen activator receptor mRNAs by binding to their regions containing the 3' UTR of their mRNAs [35]. In addition, our recent study demonstrated that resveratrol inhibits the proliferation and metastasis of colorectal cancer cells by activating TTP (unpublished data). Although the activity of resveratrol in some biological events has been reported [34], its critical molecular targets and its mechanism of action on cancer development are still unclear. Therefore, the present study aimed to investigate the mechanisms of action of resveratrol that affected the growth and development of gastric cancer cells. We demonstrated that resveratrol increases the ARE-containing mRNA decaying activity of TTP and that resveratrol has antiproliferative and anti-invasive effects, indicating suppression of tumor growth and development.

\section{Materials and Methods}

\section{Cell lines and reagents}

The gastric cancer cell lines SNU484 and SNU638 were purchased from Korean Cell Line Bank (Seoul, Korea). Cells were cultured in RPMI-1640 medium containing 10\% heat-inactivated fetal bovine serum (FBS) and $1 \%$ penicillin/streptomycin (P/S) (Capricorn Scientific, Germany) and were incubated in a humidified atmosphere containing $5 \% \mathrm{CO}_{2}$ at $37^{\circ} \mathrm{C}$. Resveratrol was procured from SigmaAldrich (34092, Sigma-Aldrich, Inc) and was dissolved in dimethyl sulfoxide (DMSO) (D2650, Sigma-Aldrich, Inc). Resveratrol treatment was given for $24 \mathrm{~h}$ to gastric cancer cells through a medium that contained $2 \%$ FBS.

\section{Plasmid construction and luciferase assay}

The pGL3/TTPp-1411 promoter [16] and several target genes of TTP in the psiCHECK2 luciferase reporter vector [psiCHECK2/3' UTR region of cIAP2 [17], E2F1 [20], LATS2 [19], and Lin28 [18]] have been already explained in our earlier studies. Cells were transfected with various kinds of plasmid DNA using jetPRIME in vitro transfection reagent (Polyplus Transfection, NY, USA). For luciferase assays, SNU484 and SNU638 cells were transfected with various plasmid DNAs and, after a day, were treated with resveratrol for $24 \mathrm{~h}$. Transfected cells were lysed with lysis buffer (Promega, WI, USA) and mixed with luciferase assay reagent, and the chemiluminescent signal was measured using a Wallac Victor 1420 multilabel counter. Firefly luciferase was normalized to renilla luciferase in each sample. All luciferase assays reported here represented at least three independent experiments, each consisting of three wells per transfection.

\section{Cell lysis and western blotting}

Proteins were extracted from SNU484 and SNU638 cells using cell lysis buffer [150 mM sodium chloride, $50 \mathrm{mM}$ Tris $\mathrm{HCl}$ ( $\mathrm{pH} 7.5), 1.5 \%$ sodium deoxycholate, $2 \mathrm{mM}$ EDTA, $1 \%(\mathrm{v} / \mathrm{v})$ Triton-X 100, 0.1\% (w/v) SDS] and protease inhibitor cocktail (Roche, Mannheim, Germany). Protein concentrations were determined using BCA protein assay (Thermo Scientific, IL, USA). Proteins were separated by SDS-PAGE and transferred onto nitrocellulose blotting membranes (GE Healthcare Life Sciences, IL, USA). They were probed with human TTP antibody (T5327, Sigma-Aldrich, Inc), $\beta$-actin (A2228, Sigma-Aldrich, Inc), and GAPDH (AbC-1001, AbClon, Korea). Immunoreactive bands were detected using the ECL detection system (Advansta, California, USA). Films were exposed at various points to avoid image saturation.

\section{Reverse transcription and quantitative real-time PCR}

Total RNA was isolated using RNAiso reagent (Takara, Japan) and was reverse transcribed to cDNA using PrimeScript RT Master Mix (Takara, Japan) following the manufacturer's protocol. cDNA was used PCR using SYBR Premix Ex Taq (Takara, Japan) and the CFX96 Optics Module (Bio-Rad, CA, USA). PCR primer pairs were as follows: TTP; 5'-CGCTACAAGACTGAGCTAT, GAGGTAGAACTTGTGACAGA-3'

and

$\beta$-actin; 5'-CCCTGGAGAAGAGCTACGAG AGGTAGTTTCGTGGATGCCA-3'. The specificity of primer pairs was confirmed by melting curve analysis and agarose gel electrophoresis. TTP expression was normalized by $\beta$-actin.

\section{RNA isolation}

Total RNA of samples was extracted using a mirVana TM miRNA isolation labeling kit (Thermo Fisher Scientific, TX, USA). Total RNA was quantified using a Nanodrop ND-1000 spectrophotometer (BioTeck, Winnoski, VT, USA).

\section{Microarray experiment and data analysis}

The microarray experiment was performed according to the manufacturer's protocols (Illumina Inc, CA, USA). Briefly, total RNA was labeled and hybridized with cyanin-3-streptavidin (GE Healthcare, NJ, USA), and bead chips were scanned with the Illumina BeadArray Reader (Illumina, CA, USA). Data were extracted using Genome Studio (Illumina, CA, USA) and normalized using quantile normalization. The expression of each gene was transformed into a $\log 2$ base. For obtaining the heat map of gene expression, Cluster and TreeView programs were used.

\section{Cell proliferation assay}

For cell proliferation assays, cells were seeded at $1 \times 10^{4}$ cells/well in triplicate in 96-well culture plates with RPMI-1640 medium (10\% FBS and $1 \% \mathrm{P} / \mathrm{S})$. Cells were incubated with different concentrations of resveratrol $(10,20,50$, or $100 \mu \mathrm{M})$ for time-dependent analysis. Then, cells were incubated with MTT ( $5 \mathrm{mg} / \mathrm{mL}$ ) (Sigma Aldrich Korea) for $90 \mathrm{~min}$ at $37^{\circ} \mathrm{C}$. After incubation for $90 \mathrm{~min}$, the medium and MTT solution were removed and the formazan product was dissolved in DMSO (D2650, Sigma-Aldrich, Inc). The absorbance of each well at $490 \mathrm{~nm}\left(\mathrm{OD}_{490}\right)$ was measured by a VICTOR3 Multilabel Reader (Perkin Elmer, MA, USA) to calculate cell counts after 12 h, 24 h, 36 $\mathrm{h}$, and $48 \mathrm{~h}$ of culture. This assay was replicated in three independent experiments with four duplicated wells.

\section{Migration and invasion assays}

The in vitro invasion assays of SNU484 and SNU638 were investigated using Boyden chambers (NeuroProbe, USA) precoated with Matrigel (BD Biosciences, USA) and Transwell inserts with collagen (C7661, Sigma-Aldrich, Inc) precoated on a membrane used for migration assay. Cells were incubated for $1 \mathrm{~h}$ at $37^{\circ} \mathrm{C}$. Bottom wells were filled with $28 \mu \mathrm{l}$ of RPMI- 1640 medium with $2 \%$ serum medium. A total of $1 \times 10^{5}$ cells per well were seeded onto the upper compartment and incubated for $24 \mathrm{~h}$ at $37^{\circ} \mathrm{C}$ and $5 \% \mathrm{CO}_{2}$. After incubation for $24 \mathrm{~h}$, cells attached to the upper surface of the filter were removed using a cotton swab and those attached to the lower surface of the filter were stained using Diff-Quik (Sysmex Co., Japan) and then counted (five fields/well). The invasion percentage showed cells passing through the membrane coated with Matrigel. The average number of stained cells was counted from five different fields of each well at $100 \times$ magnification 
Citation: Kim WT, Jin H, Lee SR, Kim SZ, Leem SH, et al. (2018) Mediation of the Anticancer Effects of Resveratrol via the Upregulation of Tristetraprolin in Gastric Cancer Cell. Med Chem (Los Angeles) 8: 029-037. doi: 10.4172/2161-0444.1000494

using an Axiovert 40 CFL inverted fluorescence microscope (Carl Zeiss Microscopy, NY, USA). Both assays were repeated three times.

\section{Colony formation assay}

SNU484 and SNU638 cells were seeded in a 12 well-plate at 2 $\times 10^{3}$ or $1 \times 10^{4}$ cells confluence and were incubated for $24 \mathrm{~h}$. Cells were treated with resveratrol in a dose-dependent manner $(5,10$, and $20 \mu \mathrm{M})$. Cells were incubated for 10 days. Fresh medium containing resveratrol was added on the third day. On the 10th day, the medium was removed from dishes and washed with PBS. Colonies were fixed and stained with methanol $(25 \% \mathrm{v} / \mathrm{v})$ containing crystal violet $(0.05 \%$ $\mathrm{w} / \mathrm{v}$ ) for $30 \mathrm{~min}$. This was followed by the removal of the time residual staining solution, and the plates were then washed with water. The dishes were dried at room temperature, and colonies were counted under a light microscope and were photographed.

\section{Statistics}

GraphPad Prism 5.0 (Graph Pad Software, CA, USA) was utilized for statistical analyses. Data are presented as mean \pm standard deviation. For statistical comparisons, P-values were determined using Student's t-test.

\section{Results}

\section{Resveratrol induced TTP expression in gastric cancer cells}

TTP expression has been shown to decrease in various cancers. Resveratrol has also been found to have anticancer effects in various cancers. Therefore, we hypothesized that resveratrol has antitumor effects via TTP mediation. To investigate whether TTP is a mediator or functions independently of the effect of resveratrol on gastric cancer cells, we treated SNU484 and SNU638 cells with different concentrations of resveratrol $(0,20$, and $50 \mu \mathrm{M})$ for $24 \mathrm{~h}$ and examined the mRNA and protein levels of TTP. As shown in Figure 1A, resveratrol significantly increased the mRNA and protein expression levels of TTP in a dose dependent manner in SNU484 and SNU638 cells (Figure 1A). We determined that the TTP expression was induced by resveratrol in a dose-dependent manner in gastric cancer cells. To further examine whether resveratrol controls the TTP expression, SNU484 and SNU638 cells were transfected with scRNA or TTP siRNA (100 nM). TTP silencing significantly decreased the relative expression of TTP mRNA levels in SNU484 and SNU638 cells (Figure 1B). To investigate whether resveratrol can rescue the TTP siRNA-induced reduction in TTP mRNA expression, we combined siRNA and resveratrol treatment in SNU484 and SNU638 cells. We further observed that TTP
A

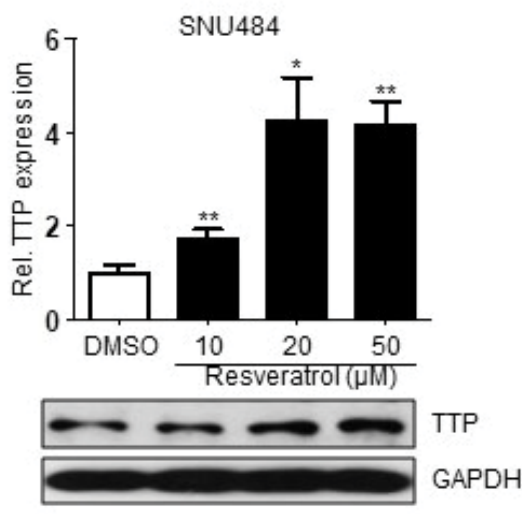

B

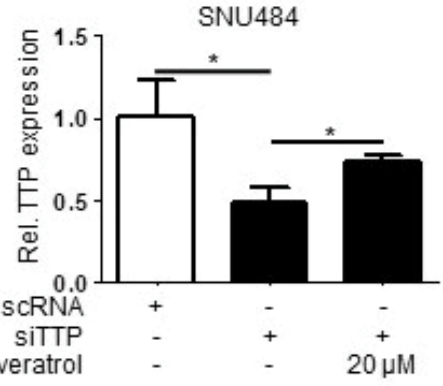

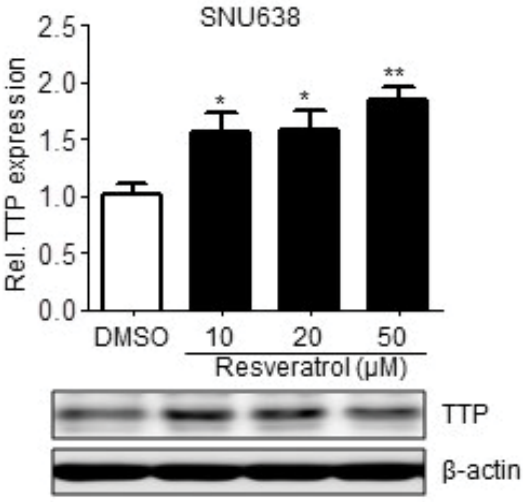

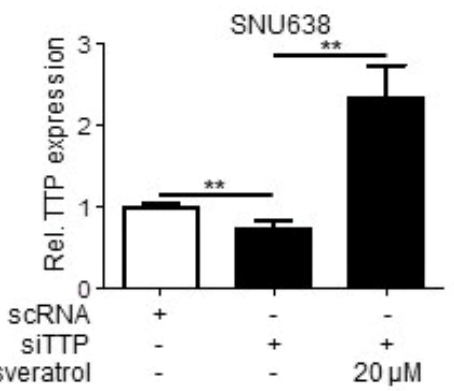

Figure 1: Resveratrol induces TTP expression in gastric cancer cells. (A) Resveratrol increased the relative mRNA and protein expression of TTP in a dosedependent manner in SNU484 and SNU638 cells. SNU484 and SNU638 cells were treated with $10 \mu \mathrm{M}, 20 \mu \mathrm{M}$, and $50 \mu \mathrm{M}$ of resveratrol in RPMI-1640 containing $2 \%$ FBS for $24 \mathrm{~h}$. TTP expression was measured using quantitative real-time PCR and western blotting. $\beta$-actin was used as a loading control in real-time PCR and western blotting. Each bar represents the mean \pm SD of three independent experiments $\left({ }^{*} P<0.05 ;{ }^{* *} P<0.01\right)$. (B) Resveratrol restored TTP expression in gastric cancer cells with reduced TTP expression using TTP siRNA. SNU484 and SNU638 cells were transfected with scRNA or TTP siRNA (100 nM). After $24 \mathrm{~h}$, DMSO or RSV $(20 \mu \mathrm{M})$ was added to SNU484 and SNU638 cells, and RNA was extracted $24 \mathrm{~h}$ later. $\beta$-actin was used as a loading control in quantitative real-time PCR. Each bar represents the mean \pm SD of three independent experiments $\left({ }^{*} P<0.05 ;{ }^{* *} \mathrm{P}<0.01 ;{ }^{* * *} \mathrm{P}<0.001\right)$. 
Citation: Kim WT, Jin H, Lee SR, Kim SZ, Leem SH, et al. (2018) Mediation of the Anticancer Effects of Resveratrol via the Upregulation of Tristetraprolin in Gastric Cancer Cell. Med Chem (Los Angeles) 8: 029-037. doi: 10.4172/2161-0444.1000494

mRNA expression was significantly restored by resveratrol treatment in SNU484 and SNU638 cells (Figure 1B). These data indicate that resveratrol significantly increases the reduced expression induced by TTP siRNA and augmented the expression levels of TTP in gastric cancer cells.

\section{Resveratrol induced TTP to regulate the downstream target gene of TTP}

We next investigated whether TTP promoter activity increased by resveratrol treatment in the reporter assay. SNU484 and SNU638 cells were transfected with the pGL3/hTTPp-1411 construct and were then treated with resveratrol. When SNU484 and SNU638 cells were treated with resveratrol for $24 \mathrm{~h}$, TTP promoter activity significantly increased (Figure 2A). As our previous studies showed that TTP reduced the expression of cIAP2, E2F1, LATS2, and Lin $28 \mathrm{mRNA}$ and inhibited the growth of various cancer cells, we further examined whether TTP regulates the stabilities of target genes (cIAP2, E2F1, LATS2, and Lin28) in gastric cancer cells. Luciferase reporter constructs containing the $3^{\prime}$ UTR of cIAP2, E2F1, LATS2, and Lin28 mRNA (psiCHECK2/cIAP2, psiCHECK2/E2F1, psiCHECK2/LATS2, and psiCHECK2/Lin28) were transfected in SNU484 and SNU638 cells. The TTP target genes construct-transfected SNU484 and SNU638 cells were treated with resveratrol, and their luciferase activity was measured after $24 \mathrm{~h}$. As shown in Figure 2B, the luciferase activities of TTP target genes (cIAP2, E2F1, LATS2, and Lin28) were significantly suppressed by resveratrol treatment in SNU484 and SNU638 cells. Resveratrol enhanced the TTP inhibitory activities of the target genes by inducing the expression of TTP in SNU484 and SNU638 cells. Therefore, our results suggested that resveratrol modulates TTP expression and promotes mRNA degradation of the target genes.

TTP was upregulated by resveratrol in the microarray experiment

To further investigate the effects of resveratrol on gastric cancer cells, we used the microarray experiment using the Illumina Bead Array Reader. We observed that multiple genes were regulated by resveratrol treatment in SNU484 cells (Figure 3). Specifically, oncogenes (TNF, E2F1, LATS1, and LIN28) and proliferation- (CTNNB1, MYC, CCND1, and KRAS), angiogenesis- (VEGFA, VEGFB, and VEGFC), and metastasis- (FOXM1, PTGS1, PTGS1, and TWIST1) related genes were downregulated by resveratrol treatment in SNU484 cells. Importantly, TTP gene expression significantly increased with resveratrol treatment in SNU484 cells. This result was consistent with our data shown in Figure 1 showing that mRNA and protein levels of TTP increased after resveratrol treatment in gastric cancer cells. Although the luciferase activities of the TTP target genes (cIAP2, E2F1, LATS2, and Lin28) significantly decreased by resveratrol treatment in SNU484 and SNU638 cells, the microarray experiment revealed that the luciferase activities of the TTP target genes slightly decreased by resveratrol treatment (Figure 3). Therefore, these results suggested that resveratrol induces the upregulation of TTP and their target genes in gastric cancer cells.
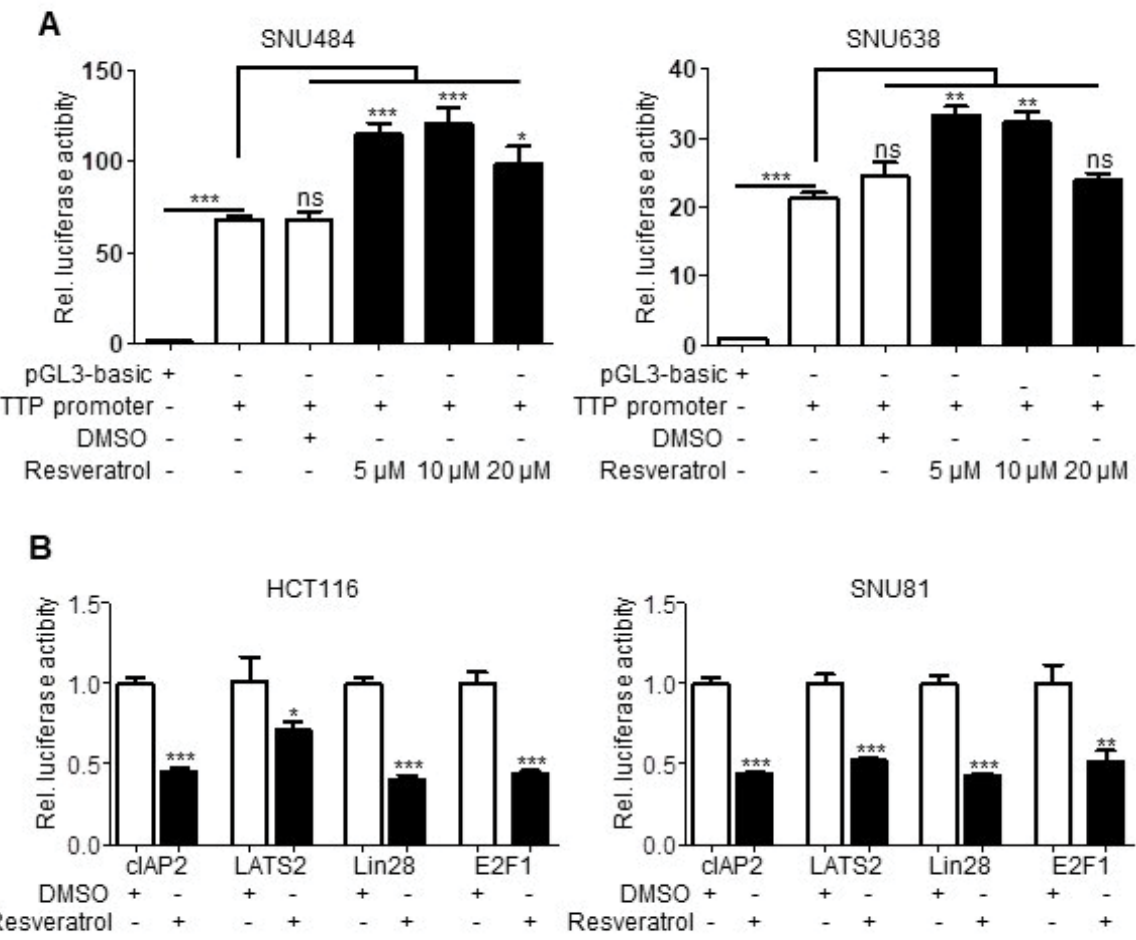

Figure 2: Resveratrol induces TTP promoter activities in SNU484 and SNU638 cells. (A) SNU484 and SNU638 cells were transfected with pGL3/TTPp-1411 containing the TTP promotor $(-1343$ to +68$)$ for $24 \mathrm{~h}$ and were then treated with resveratrol in a dose-dependent manner for $24 \mathrm{~h}$. After treatment with RSV for $24 \mathrm{~h}$, luciferase activity was measured. Expression levels obtained from pGL3-basic- (empty vector) transfected cells without resveratrol treatment were set to 1. Each bar represents the mean \pm SD of three independent experiments. (B) Resveratrol induced the mRNA decaying activity of TTP. SNU484 and SNU638 cells were transfected with psiCHECK2-cIAP2, LATS2, Lin28 and E2F1 3' UTR. At $24 \mathrm{~h}$ post transfection, cells were treated with resveratrol at a concentration of 20 $\mu \mathrm{M}$ for $24 \mathrm{~h}$, and luciferase activity was measured. Renilla luciferase activity was normalized to firefly activity. Luciferase activity obtained from psiCHECK2-cIAP2, LATS2, Lin28, MDM2 and E2F1 3' UTR-transfected and DMSO-treated cells was set to 1. Each bar represents the mean \pm SD of three independent experiments $\left({ }^{*} \mathrm{P}<0.05 ;{ }^{*} \mathrm{P}<0.01 ;{ }^{* *} \mathrm{P}<0.001\right)$. 
Citation: Kim WT, Jin H, Lee SR, Kim SZ, Leem SH, et al. (2018) Mediation of the Anticancer Effects of Resveratrol via the Upregulation of Tristetraprolin in Gastric Cancer Cell. Med Chem (Los Angeles) 8: 029-037. doi: 10.4172/2161-0444.1000494

\section{Resveratrol inhibited the proliferation of gastric cancer cells}

Given the tumor-suppressive potential of resveratrol, we investigated the biological functions of resveratrol in regulating the growth of gastric cancer cells. As shown in Figure 4A, resveratrol significantly suppressed the proliferation of SNU484 and SNU638 cells in a time- and a dose-dependent manner at $48 \mathrm{~h}$. In addition, the colony formation assay showed that colony formation by gastric cancer cells was significantly inhibited by the increase in resveratrol concentration (Figure 4B). To further test whether the resveratrol-mediated inhibition of cell proliferation correlated with TTP expression, SNU484 and SNU638 cells were transfected with scRNA or TTP siRNA $(100 \mathrm{nM})$ and had undergone resveratrol treatment. As shown in Figure 4C, TTP siRNA, but not scRNA, decreased the inhibitory effects of resveratrol on the growth of SNU484 and SNU638 cells. Taken together, these results suggest that resveratrol inhibits gastric cancer cell growth mediated through TTP and that resveratrol induces the expression of TTP in gastric cancer cells.

\section{Resveratrol inhibited invasiveness and migratory ability in gastric cancer cells}

We further tested whether resveratrol regulates migration and metastasis in gastric cancer cells. To confirm cell invasion and migration, the invasiveness and migratory ability of SNU484 and SNU638 cells treated with resveratrol were measured by the invasion and migration assays, respectively. The invasion assay was performed using Boyden chambers precoated with Matrigel. As shown in Figure $5 \mathrm{~A}$, resveratrol significantly suppressed the invasiveness of SNU484 and SNU638 cells in a dose-dependent manner. In the migration assay, the migratory ability of SNU484 and SNU638 cells were significantly inhibited in a dose-dependent manner (Figure 5B). These results suggest that resveratrol inhibits the invasion and metastasis of gastric cancer cells.

\section{Discussion}

Gastric cancer is one of the most common cancers in Asian countries including South Korea. To date, the well-known therapeutic options available for gastric cancer include chemotherapy, radiotherapy, and surgical procedures; however, these are associated with side effects. Hence, discovering a secure and an effective anticancer treatment is currently drawing considerable attention. Over recent years, natural compounds with potential anticancer effects have been acknowledged for their possible role as chemopreventive agents. Resveratrol is a natural polyphenolic flavonoid compound produced by many commonly consumed plants including grapes, apples, raspberries, blueberries, plums, and peanuts $[30,36]$. It has multiple healthpromoting effects, mainly against oxidative stress, inflammation, and apoptosis. Furthermore, it decreases migration and invasion by regulating multiple mechanisms in gastric cancer cells [37-43]. Yang et al. have shown that resveratrol inhibited the growth of gastric cancer by inducing G1-phase arrest and senescence in a sirt1-dependent manner [41]. However, the mechanism of the antitumor effects of resveratrol on gastric cancer has not been clearly understood. Investigating the precise mechanism will help our understanding of the experimental and theoretical bases for the application of resveratrol in treating gastric cancer.

TTP is an ARE-binding protein that regulates the stability of mRNAs and involved in the post-transcriptional regulation of many proinflammatory cytokines and transcription factors [44]. TTP expression was suppressed in various cancers. TTP may act as a tumor suppressor by inhibiting its target oncogenes [45]. Further,
TTP upregulation has been found to suppress the development of inflammation and many cancers [34,46]. Recent studies have demonstrated that TTP expression changed with resveratrol treatment in some cancers $[34,35]$. Using breast cancer cells, Li et al. have reported that resveratrol suppressed the growth of cancer cells by increasing TTP expression and changing the expression of the downstream effectors of TTP including VEGF, COX-2, and iNOS [34]. Ryu et al. have reported that resveratrol suppressed cell growth and induced apoptosis by increasing TTP expression in human glioma cells [35]. Moreover, our recent study showed that TTP upregulation by resveratrol treatment suppressed the proliferation and metastasis of colorectal cancer cells (unpublished data). Therefore, it is valuable to examine whether resveratrol acts against cancers through the engagement of TTP. In the present study, we explored whether TTP is regulated by resveratrol in gastric cancer cells. We also investigated the molecular mechanisms behind TTP regulation that governed cell proliferation and migration/ invasion in gastric cancer cells. We found that resveratrol increased the expression of TTP in a dose-dependent manner in SNU484 and SNU638 cells (Figure 1A). The relative TTP mRNA levels showed a significant decrease by TTP silencing in SNU484 and SNU638 cells (Figure 1B). TTP mRNA expression was significantly restored by resveratrol treatment in SNU484 and SNU638 cells. In addition, siRNA-induced TTP inhibition attenuated the effects of resveratrol on cell growth. These results suggest that TTP is involved in the effect of resveratrol on the inhibition of gastric cancer cell growth and indicate that resveratrol significantly increased the reduced expression induced by TTP siRNA. Our observations are in agreement with those made in earlier studies wherein TTP expression was increased by resveratrol treatment in glioma and breast cancer cells $(34,35)$. These results suggest that resveratrol increases TTP expression in gastric cancer cells. We next investigated whether TTP promoter activity would be regulated by resveratrol treatment. TTP promoter activity was significantly increased by resveratrol treatment after $24 \mathrm{~h}$ in SNU484 and SNU638 cells (Figure 2A). In previous studies, we found that TTP downregulated many oncogenes, such as cIAP2, E2F1, LATS2, Lin28, and VEGF, in various cancer cells $[16,17,19,20]$. Therefore, we further studied whether TTP controls the stabilities of target genes such as

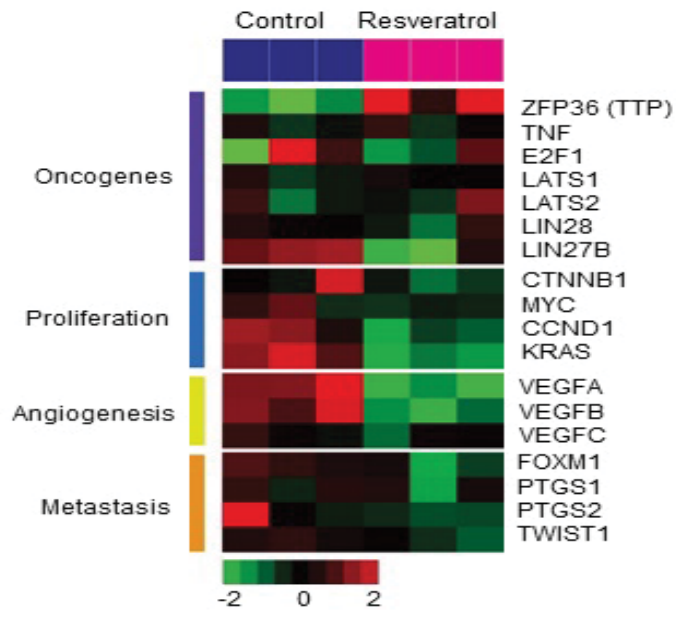

Figure 3: Hierarchical clustering analysis. The effects of resveratrol on oncogenes and proliferation-, angiogenesis-, and metastasis-related genes in the heat map of SNU484 cells. Data are shown in the matrix format, with rows revealing the individual gene and columns revealing each sample. Red and green indicate increased and decreased gene expression levels, respectively, as indicated in the scale bar (log2 transformed). 
Citation: Kim WT, Jin H, Lee SR, Kim SZ, Leem SH, et al. (2018) Mediation of the Anticancer Effects of Resveratrol via the Upregulation of Tristetraprolin in Gastric Cancer Cell. Med Chem (Los Angeles) 8: 029-037. doi: 10.4172/2161-0444.1000494
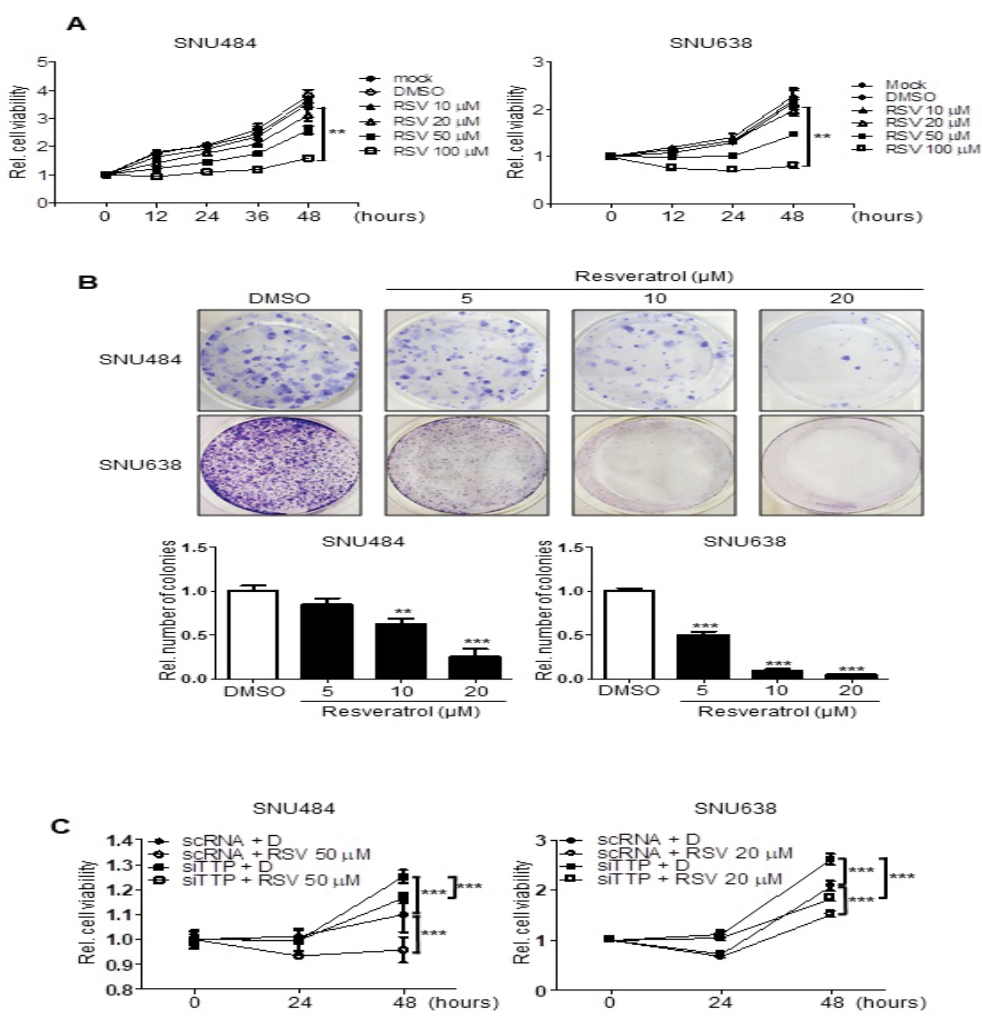

Figure 4: TTP mediates the anticancer effects of resveratrol on gastric cancer cells. (A) Resveratrol suppressed the tumor progression of gastric cancer cells. Cell viability of cancer cells decreased after resveratrol in the MTT assay. SNU484 and SNU638 cells treated with resveratrol in a dose-dependent manner were seeded at $1 \times 10^{4}$ cells per well in 96-well plates. Data represent the mean \pm SD of three independent experiments $\left({ }^{* *} \mathrm{P}<0.001\right)$. (B) Clonogenic assay performed in SNU484 and SNU638 cells treated with resveratrol in a dose-dependent manner for determining proliferation rates. SNU484 and SNU638 cells were seeded at $1 \times 10^{3}$ cells per well in 12-well plates. Next, resveratrol was added at the indicated concentration for $48 \mathrm{~h}$ and after that was replaced with fresh medium. Cells were stained with $0.05 \%$ crystal violet after incubation for 10 days. Data represent the mean $\pm S D$ of three independent experiments ( $\left.{ }^{*} P<0.05 ;{ }^{* *} P<0.01 ;{ }^{* * *} P<0.001\right)$. (C) Cell proliferation rates were measured using the MTT assay. SNU484 and SNU638 cells were transfected with scRNA or TTP siRNA (100 nM). After $24 \mathrm{~h}$, DMSO or RSV $(20 \mu \mathrm{M})$ was added to SNU484 and SNU638 cells, and the MTT assay was performed $48 \mathrm{~h}$ later. Cell survival relative to that of the control (\%) is expressed as the mean \pm SD of three independent experiments $\left({ }^{* *} P<0.001\right)$.

cIAP2, E2F1, LATS2, and Lin28 in gastric cancer cells. The luciferase activities of cIAP2, E2F1, LATS2, and Lin28 significantly decreased by resveratrol treatment in SNU484 and SNU638 cells (Figure 2B). In the microarray experiment, TTP was upregulated and target genes (E2F1, LATS2, and Lin28) were downregulated by resveratrol treatment in gastric cancer cells (Figure 3). These findings are in agreement with those obtained in earlier studies that demonstrated a decline in the stability of E2F1 and Lin28 mRNAs by TTP overexpression [20,47] and TTP regulated the stability of cIAP2 and LATS 2 mRNAs by binding to the $3^{\prime}$ UTR of cIAP2 mRNA or promoting let-7 biogenesis $[19,48]$. These results indicate that cIAP2, E2F1, LATS2, and Lin28 are physiological targets of TTP and that TTP directly regulated cell death through the decay of cIAP2, E2F1, LATS2, and Lin28 mRNAs. Therefore, resveratrol enhances the TTP inhibitory activities of the target genes by inducing the expression of TTP in gastric cancer cells.

The present study also demonstrated that resveratrol significantly inhibited the viability of SNU484 and SNU638 cells in a time- and dosedependent manner (Figure 4A). Moreover, resveratrol suppressed colony formation by gastric cancer cells in a dose-dependent manner (Figure 4B). In addition, the inhibition of TTP by siRNA attenuated the effects of resveratrol on cell growth (Figure 4C). Moreover, resveratrol reduced the invasion and metastasis of gastric cancer cells (Figure 5). Hence, the expression of resveratrol-potentiated TTP induced cell death in gastric cancer cells. These results suggest that TTP mediates the inhibition of cell growth and metastasis effects of resveratrol on gastric cancer cells. TTP has been reported to be involved in numerous biological roles, including cell growth, differentiation, apoptosis, metastasis, and tumorigenesis, in various cancer cells [49-53]. In gastric cancer, TTP has been reported to suppress the progression of gastric cancer through IL-33 suppression [44]. Therefore, our results suggest that TTP directly regulated cell death through the decay of cIAP2, E2F1, LATS2, and Lin28 mRNAs in gastric cancer cells. However, further studies are required to characterize the precise mechanism mediating TTP expression by resveratrol on its target genes in gastric cancer cells.

\section{Conclusion}

In conclusion, our results provide strong evidence that resveratrol is an effective inhibitor of gastric cancer cell growth and metastasis by increasing TTP expression. The observed antiproliferative and antimetastatic effects of resveratrol may be due to inhibit cIAP2, E2F1, 
Citation: Kim WT, Jin H, Lee SR, Kim SZ, Leem SH, et al. (2018) Mediation of the Anticancer Effects of Resveratrol via the Upregulation of Tristetraprolin in Gastric Cancer Cell. Med Chem (Los Angeles) 8: 029-037. doi: 10.4172/2161-0444.1000494

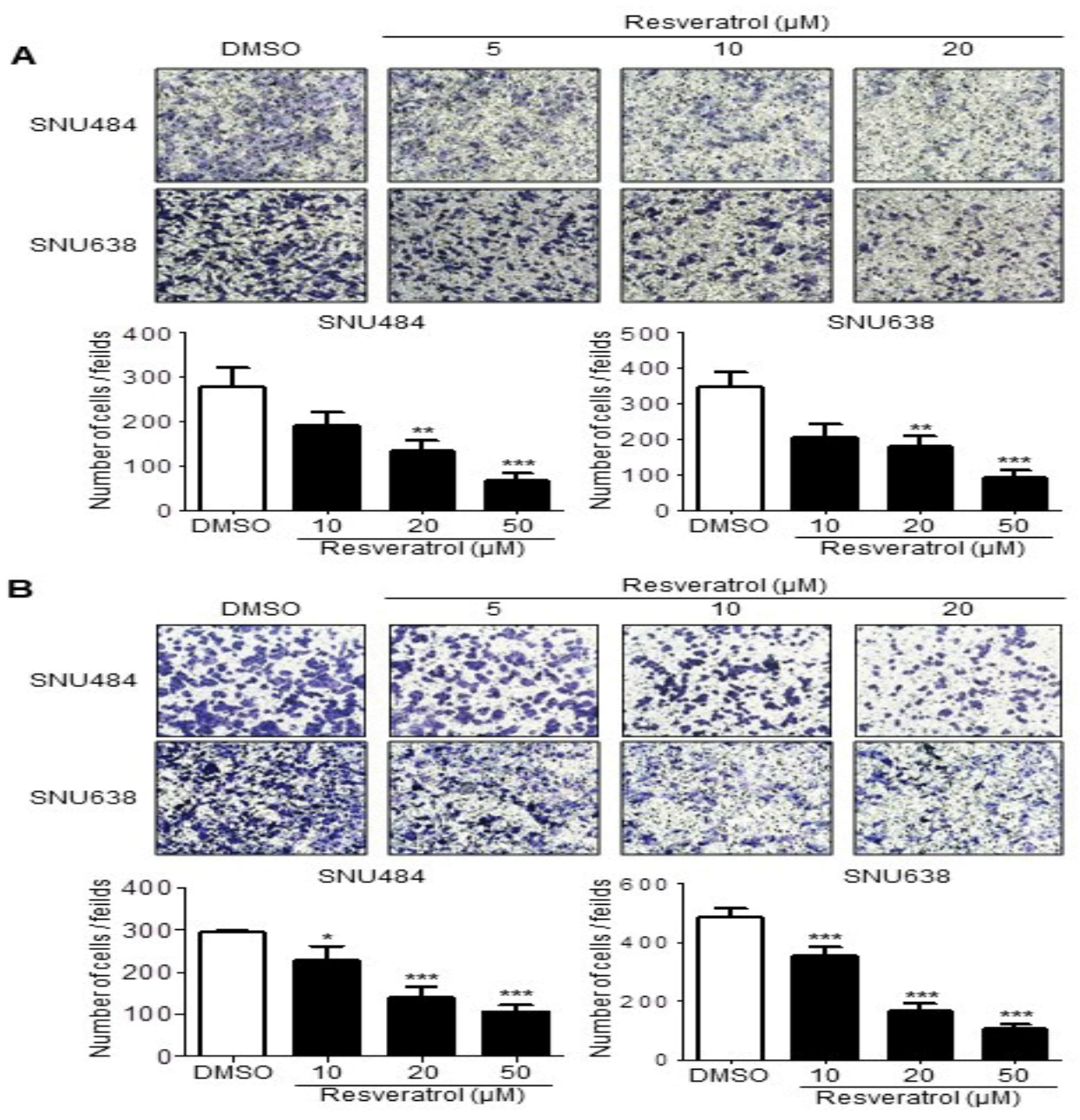

Figure 5: Resveratrol inhibits invasion and migration in gastric cancer cells. (A) The in vitro invasive properties of SNU484 and SNU638 cells were studied using Boyden chambers precoated with Matrigel or collagen and incubated for $1 \mathrm{~h}$ at $37^{\circ} \mathrm{C}$. Cells were seeded at $4 \times 10^{5}$ cells per well in 6 well-plates and were treated with RSV for $24 \mathrm{~h}$. Next, cells were collected and reseeded at $5 \times 10^{4}$ cells per well in the chamber. Bottom wells were filled with $28 \mu \mathrm{ll}$ of medium containing $2 \%$ serum medium. After incubation for $24 \mathrm{~h}$, cells attached the upper surface of the filter were removed using a cotton swab and those attached to the lower surface of the filter were stained using Diff-Quik and counted (five fields/well). Invading cells were visualized by staining membranes using Diff-Quick, and they were then counted (five fields/well). Graphical representation of the results seen in SNU484 and SNU638 cells ( $\left.{ }^{* *} P<0.01 ;{ }^{* * *} P<0.001\right)$. (B) SNU484 and SNU638 cells were treated with resveratrol, and the decreased rate of invasive and migratory cells was determined. TTP potently suppressed invasion and migration by gastric cancer cells after treatment with resveratrol in a dose-dependent manner as seen in the Boyden chambers. Data represent the mean \pm SD of three independent experiments $\left({ }^{* *} \mathrm{P}<0.01 ;{ }^{* * *} \mathrm{P}<0.001\right)$.

LATS2, and Lin28 mRNAs by binding to their $3^{\prime}$ UTRs) via TTP activation. These findings that TTP engages the anticancer effects of resveratrol consolidate our understanding of the mechanism through which resveratrol functions against cancers.

\section{Acknowledgements}

No author had a conflict of interest with respect to the reported data. This research was supported by "Research Base Construction Fund Support Program" funded by Chonbuk National University in 2017, by the Basic Science Research Program (2015R1C1A2A01054054), by grants from the Medical Research Center Program (NRF-2017R1A5A2015061) through the National Research Foundation, which is funded by the Korean government (MSIP), and by a grant of the Korea Health Technology R\&D Project through the Korea Health Industry Development Institute, funded by the Ministry of Health \& Welfare, Republic of Korea (HI16C1866).

\section{Conflict of Interest}

The authors disclose no potential conflict of interest.

\section{References}

1. Pinheiro Ddo R, Ferreira WA, Barros MB, Araujo MD, Rodrigues-Antunes $S$ (2014) Perspectives on new biomarkers in gastric cancer: diagnostic and prognostic applications. World J Gastroenterol 20: 11574-11585.

2. Parkin DM, Bray F, Ferlay J, Pisani P (2005) Global cancer statistics, 2002. CA Cancer J Clin 55: 74-108.

3. Ahn HS, Jeong SH, Son YG (2014) Effect of neoadjuvant chemotherapy on postoperative morbidity and mortality in patients with locally advanced gastric cancer. Br J Surg 101: 1560-1565.

4. Torre LA, Bray F, Siegel RL, Ferlay J, Lortet-Tieulent J, et al. (2015) Global cancer statistics, 2012. CA Cancer J Clin 65: 87-108.

5. Torre LA, Siegel RL, Ward EM, Jemal A (2016) Global Cancer Incidence and Mortality Rates and Trends--An Update. Cancer Epidemiol Biomarkers Prev 25: 16-27.

6. Lai WS, Thompson MJ, Blackshear PJ (1998) Characteristics of the intron 
Citation: Kim WT, Jin H, Lee SR, Kim SZ, Leem SH, et al. (2018) Mediation of the Anticancer Effects of Resveratrol via the Upregulation of Tristetraprolin in Gastric Cancer Cell. Med Chem (Los Angeles) 8: 029-037. doi: 10.4172/2161-0444.1000494

involvement in the mitogen-induced expression of Zfp-36. J Biol Chem 273: 506-517.

7. Lai WS, Thompson MJ, Taylor GA, Liu Y, Blackshear PJ (1995) Promoter analysis of Zfp-36, the mitogen-inducible gene encoding the zinc finger protein tristetraprolin. J Biol Chem 270: 25266-25272.

8. Taylor GA, Thompson MJ, Lai WS, Blackshear PJ (1995) Phosphorylation of tristetraprolin, a potential zinc finger transcription factor, by mitogen stimulation in intact cells and by mitogen-activated protein kinase in vitro. J Biol Chem 270 : $13341-13347$.

9. Lai WS, Carballo E, Thorn JM, Kennington EA, Blackshear PJ (2000) Interactions of $\mathrm{CCCH}$ zinc finger proteins with mRNA. Binding of tristetraprolinrelated zinc finger proteins to Au-rich elements and destabilization of mRNA. J Biol Chem 275: 17827-17837.

10. Lai WS, Blackshear PJ (2001) Interactions of $\mathrm{CCCH}$ zinc finger proteins with mRNA: tristetraprolin-mediated AU-rich element-dependent mRNA degradation can occur in the absence of a poly(A) tail. J Biol Chem 276: 23144-23154.

11. Blackshear PJ (2002) Tristetraprolin and other $\mathrm{CCCH}$ tandem zinc-finger proteins in the regulation of mRNA turnover. Biochem Soc Trans 30: 945-952.

12. Lai WS, Kennington EA, Blackshear PJ (2002) Interactions of $\mathrm{CCCH}$ zinc finger proteins with mRNA: non-binding tristetraprolin mutants exert an inhibitory effect on degradation of AU-rich element-containing mRNAs. J Biol Chem 277: 9606-9613.

13. Phillips RS, Ramos SB, Blackshear PJ (2002) Members of the tristetraprolin family of tandem $\mathrm{CCCH}$ zinc finger proteins exhibit CRM1-dependent nucleocytoplasmic shuttling. J Biol Chem 277: 11606-11613.

14. Stoecklin G, Gross B, Ming XF, Moroni C (2003) A novel mechanism of tumor suppression by destabilizing AU-rich growth factor mRNA. Oncogene 22: 35543561.

15. Stoecklin G, Ming XF, Looser R, Moroni C (2000) Somatic mRNA turnover mutants implicate tristetraprolin in the interleukin-3 mRNA degradation pathway. Mol Cell Biol 20: 3753-3763.

16. Lee JY, Kim HJ, Yoon NA (2013) Tumor suppressor p53 plays a key role in induction of both tristetraprolin and let-7 in human cancer cells. Nucleic Acids Res 41: 5614-5625.

17. Kim CW, Kim HK, Vo MT (2010) Tristetraprolin controls the stability of clAP2 mRNA through binding to the 3'UTR of clAP2 mRNA. Biochem Biophys Res Commun 400: 46-52.

18. Kim CW, Vo MT, Kim HK (2012) Ectopic over-expression of tristetraprolin in human cancer cells promotes biogenesis of let- 7 by down-regulation of Lin28. Nucleic Acids Res 40: 3856-3869.

19. Lee HH, Vo MT, Kim HJ (2010) Stability of the LATS2 tumor suppressor gene is regulated by tristetraprolin. J Biol Chem 285: 17329-17337.

20. Lee HH, Lee SR, Leem SH (2014) Tristetraprolin regulates prostate cancer cell growth through suppression of E2F1. J Microbiol Biotechnol 24: 287-294.

21. Bollmann F, Wu Z, Oelze M (2014) Endothelial dysfunction in tristetraprolindeficient mice is not caused by enhanced tumor necrosis factor-alpha expression. J Biol Chem 289: 15653-15665.

22. Qiu LQ, Lai WS, Bradbury A, Zeldin DC, Blackshear PJ (2015) Tristetraprolin (TTP) coordinately regulates primary and secondary cellular responses to proinflammatory stimuli. J Leukoc Biol 97: 723-736.

23. Sanduja S, Blanco FF, Young LE, Kaza V, Dixon DA (2012) The role of tristetraprolin in cancer and inflammation. Front Biosci (Landmark Ed) 17: 174188.

24. Hardle L, Bachmann M, Bollmann F (2015) Tristetraprolin regulation of interleukin-22 production. Sci Rep 5: 15112

25. Dong Z (2003) Molecular mechanism of the chemopreventive effect of resveratrol. Mutat Res 523-524: 145-150.

26. Brisdelli F, D'Andrea G, Bozzi A (2009) Resveratrol: a natural polyphenol with multiple chemopreventive properties. Curr Drug Metab 10: 530-546.

27. Maru GB, Hudlikar RR, Kumar G, Gandhi K, Mahimkar MB (2016) Understanding the molecular mechanisms of cancer prevention by dietary phytochemicals: From experimental models to clinical trials. World J Biol Chem 7: 88-99.

28. Wang W, Zhang L, Chen T (2017) Anticancer Effects of Resveratrol-Loaded Solid Lipid Nanoparticles on Human Breast Cancer Cells. Molecules 22: 1814.

29. Wakimoto R, Ono M, Takeshima M, Higuchi T, Nakano S (2017) Differential Anticancer Activity of Pterostilbene Against Three Subtypes of Human Breast Cancer Cells. Anticancer Res 37: 6153-6159.

30. Pan J, Shen J, Si W (2017) Resveratrol promotes MICA/B expression and natural killer cell lysis of breast cancer cells by suppressing c-Myc/miR-17 pathway. Oncotarget 8: 65743-65758.

31. Pistollato F, Calderon Iglesias R, Ruiz R (2017) The use of natural compounds for the targeting and chemoprevention of ovarian cancer. Cancer Lett 411: 191 200 .

32. McCubrey JA, Abrams SL, Lertpiriyapong K (2017) Effects of berberine curcumin, resveratrol alone and in combination with chemotherapeutic drugs and signal transduction inhibitors on cancer cells-Power of nutraceuticals. Adv Biol Regul.

33. McCubrey JA, Lertpiriyapong K, Steelman LS (2017) Effects of resveratrol, curcumin, berberine and other nutraceuticals on aging, cancer development, cancer stem cells and microRNAs. Aging (Albany NY) 9: 1477-1536.

34. Li C, Tang C, He G (2016) Tristetraprolin: a novel mediator of the anticancer properties of resveratrol. Genet Mol Res 15: gmr.15027213.

35. Ryu J, Yoon NA, Seong H (2015) Resveratrol Induces Glioma Cell Apoptosis through Activation of Tristetraprolin. Mol Cells 38: 991-997.

36. Szliszka E, Krol W (2011) The role of dietary polyphenols in tumor necrosis factor-related apoptosis inducing ligand (TRAIL)-induced apoptosis for cancer chemoprevention. Eur J Cancer Prev 20: 63-69.

37. Xu J, Liu D, Niu H (2017) Resveratrol reverses Doxorubicin resistance by inhibiting epithelial-mesenchymal transition (EMT) through modulating PTEN/ Akt signaling pathway in gastric cancer. J Exp Clin Cancer Res 36: 19.

38. Jing X, Cheng W, Wang S, Li P, He L (2016) Resveratrol induces cell cycle arrest in human gastric cancer MGC803 cells via the PTEN-regulated PI3K/Akt signaling pathway. Oncol Rep 35: 472-478.

39. Gao Q, Yuan Y, Gan HZ, Peng Q (2015) Resveratrol inhibits the hedgehog signaling pathway and epithelial-mesenchymal transition and suppresses gastric cancer invasion and metastasis. Oncol Lett 9: 2381-2387.

40. Liu ML, Zhang SJ (2014) Effects of resveratrol on the protein expression of survivin and cell apoptosis in human gastric cancer cells. J BUON 19: 713-717.

41. Yang Q, Wang B, Zang W (2013) Resveratrol inhibits the growth of gastric cancer by inducing $\mathrm{G} 1$ phase arrest and senescence in a Sirt1-dependent manner. PLoS ONE 8: e70627.

42. Wang Z, Li W, Meng X, Jia B (2012) Resveratrol induces gastric cancer cell apoptosis via reactive oxygen species, but independent of sirtuin1. Clin Exp Pharmacol Physiol 39: 227-232.

43. Signorelli P, Munoz-Olaya JM, Gagliostro V, Casas J, Ghidoni R, et al. (2009) Dihydroceramide intracellular increase in response to resveratrol treatment mediates autophagy in gastric cancer cells. Cancer Lett 282: 238-243.

44. Deng K, Wang H, Shan T (2016) Tristetraprolin inhibits gastric cancer progression through suppression of IL-33. Sci Rep 6: 24505.

45. Brennan SE, Kuwano Y, Alkharouf N, Blackshear PJ, Gorospe M, et al. (2009) The mRNA-destabilizing protein tristetraprolin is suppressed in many cancers, altering tumorigenic phenotypes and patient prognosis. Cancer Res 69: 51685176 .

46. Yin S, Deng W, Zheng H, Zhang Z, Hu L, et al. (2009) Evidence that the nonsense-mediated mRNA decay pathway participates in $\mathrm{X}$ chromosome dosage compensation in mammals. Biochem Biophys Res Commun 383: 378382 .

47. Cha HJ, Lee HH, Chae SW (2011) Tristetraprolin downregulates the expression of both VEGF and COX-2 in human colon cancer. Hepatogastroenterology 58 790-795. 
Citation: Kim WT, Jin H, Lee SR, Kim SZ, Leem SH, et al. (2018) Mediation of the Anticancer Effects of Resveratrol via the Upregulation of Tristetraprolin in Gastric Cancer Cell. Med Chem (Los Angeles) 8: 029-037. doi: 10.4172/2161-0444.1000494

48. Lee HH, Son YJ, Lee WH (2010) Tristetraprolin regulates expression of VEGF and tumorigenesis in human colon cancer. Int $\mathrm{J}$ Cancer 126: 1817-1827.

49. Yoon NA, Jo HG, Lee UH (2016) Tristetraprolin suppresses the EMT through the down-regulation of Twist1 and Snail1 in cancer cells. Oncotarget 7: 8931-8943.

50. Wei ZR, Liang C, Feng D (2016) Low tristetraprolin expression promotes cell proliferation and predicts poor patients outcome in pancreatic cancer. Oncotarget 7: 17737-17750.
51. Xu L, Ning H, Gu L (2015) Tristetraprolin induces cell cycle arrest in breast tumor cells through targeting AP-1/c-Jun and NF-kappaB pathway. Oncotarget 6: $41679-41691$

52. Suswam E, Li Y, Zhang X (2008) Tristetraprolin down-regulates interleukin-8 and vascular endothelial growth factor in malignant glioma cells. Cancer Res 68: 674-682.

53. Zeng B, Zhu D, Su Z, Li Z, Yu Z (2016) Tristetraprolin exerts tumor suppressive functions on the tumorigenesis of glioma by targeting IL-13. Int Immunopharmacol 39: 63-70. 